\title{
Una metodología para analizar la relación costo-volumen-utilidades en las empresas hoteleras.
}

\section{José Marsano Delgado}

La gestión empresarial en el mismo negocio de las empresas hoteleras, cuando las mismas alcanzan un movimiento económico y financiero significativo, implica por parte de la gerencia, el medir constantemente sus puntos de equilibrio, dado que estas empresas dependen de un mercado en el que las mayorias de las variables a las que se enfrentan son de naturaleza exógenas y por lo tanto poco pueden hacer por ejemplo en el control del flujo de turistas o visitantes al pais, si estos dependen de períodos vacacionales o de condiciones socio politicas poco favorables y que implican un clima de incertidumbre y intranquilidad en el país.

En el caso del turismo receptor peruano, el grado de ocupación de la infraestructura hotelera depende básicamente del flujo de turistas que provienen en un gran porcentaje de países pertenecientes al hemisferio norte y de la afluencia de visitantes que vienen al país a efectos de realizar negocios.

Las empresas hoteleras deben contar con un instrumental económico, financiero y contable, como el que se propone en el presente artículo a efectos de tomar las medidas mas adecuadas para optimizar el manejo económico de las mismas.

\begin{abstract}
Variabilidad del costo
El análisis del estudio de la relación costo-volumen-utilidad depende de una adecuada segregación de los costos de acuerdo a su variabilidad. Los costos por lo general poca veces encajan dentro de las teóricas definiciones de los costos totalmente variables o totalmente fijos y de ello se debe inferir que los costos variables no son siempre perfectamente variables ni los costos fijos son siempre perfectamente fijos.
\end{abstract}

Los costos fijos son solo relativamente fijos y en cortos períodos de tiempo. A la larga los costos fijos se convierten en costos variables. Aún en el lapso de breves períodos el reemplazo de equipos y maquinarias $u$ otros activos por substitutos más caros aumenta los costos fijos.

Los costos semivariables son parcialmente fijos y parcialmente variables.

Algunos costos clasificados como variables tienen un comportamiento curvilineal más que lineal. Esto implica que varian de manera distinta en los distintos niveles de rendimiento o producción. A medida que aumenta los servicios, los costos de energía eléctrica tienden a aumentar a un ritmo declinante, mientras que los costos de reparación siguen un curso inverso. 


\section{Determinación de la variabilidad de los costos.}

Para determinar la variabilidad de los costos es imprescindible determinar todos aquellos elementos que intervienen en el costo de la producción de un determinado servicio. Los registros contables suelen ser la base de partida. Después de agregar los elementos de costos individuales, es necesario correlacionarlos con una base variable. La mayoría de los costos al menos inicialmente, no se traducen en estrechas relaciones con una base variable. Sin perjuicio de lo antes expuesto, la variable ventas es la que más se utiliza a efectos de determinar la variabilidad de todos los costos. Otras metodologias que se utilizan son los diagramas de dispersión y los métodos matemáticos, los mismos que permiten efectuar un análisis más preciso de costos históricos.

Es conveniente hacer notar al lector que el analista que desea proyectar o interpretar datos de costo-volumen-utilidades enfrentará ciertas limitaciones que suelen presentarse, co- mo por ejemplo el cambio de la futura conducta de los consumidores, las mismas que no obedecen a variables endógenos de las empresas hoteleras, sino a variables exógenos y ello conlleva a correr un serios riesgos en las proyecciones. En tal sentido es recomendable analizar cada variable sujeta a proyección, teniendo no solo en cuenta los factores cuantitativos, que en una determinada serie cuanto mayor sea la misma en términos de tiempo, esta arrojará un mayor grado de confiabilidad, pero no es menos cierto que la misma debe también ser sometida a un análisis sumamente riguroso desde el punto cualitativo, y para ello el analista debe de estar bien informado de las tendencias de los mercados tanto turísticos, como de visitantes.

\section{Procesamiento de los datos: costo- volumen-utilidades}

Una vez que han sido determinados los elementos fijos y variables de cada costo, ya se está en condiciones de poder elaborarse un pronóstico de utilidades para distintos niveles de operación como se explica en el cuadro número uno.

\section{Cuadro 1 Pronóstico de ingreso Bruto a Diferentes Niveles de ocupación}

Grado de ocupabilidad

Ventas Nuevos Soles

Costos Variables

Materiales

Mano de obra directa

Gastos generales de senicio

Supervisión

Trabajos de oficina

Manejo de insumos

Bonificaciones

Beneficios sociales

Reparaciones

Suministros

Servicios públicos

Lavanderia

Otros

Gastos de ventas y administrativos

Total Costos Variables

Margen disponible para costos

fijos y utilidades

Gastos generales del hotel

Seguridad

Gastos de venta y administrativos

Total Costos Fijos

Utilidad

\begin{tabular}{|c|c|c|c|c|c|}
\hline $\begin{array}{c}50 \% \\
200,000 \\
\end{array}$ & $\begin{array}{c}60 \% \\
240,000 \\
\end{array}$ & $\begin{array}{c}70 \% \\
280,000 \\
\end{array}$ & $\begin{array}{c}80 \% \\
320,000\end{array}$ & $\begin{array}{c}90 \% \\
360,000\end{array}$ & $\begin{array}{c}100 \% \\
400,000 \\
\end{array}$ \\
\hline 60,000 & 72,000 & 84,000 & 96,000 & 108,000 & 120,000 \\
\hline 20,000 & 24,000 & 28,000 & 32,000 & 36,000 & 40,000 \\
\hline 3,000 & 3,600 & 4,200 & 0 & 5,400 & 6,000 \\
\hline 400 & 480 & 560 & 640 & 720 & 800 \\
\hline 200 & 240 & 280 & 320 & 360 & 400 \\
\hline 400 & 480 & 560 & 640 & 720 & 800 \\
\hline 2,000 & 2,400 & 2,800 & 3,200 & 3,600 & 4,000 \\
\hline 3,000 & 3,600 & 4,200 & 4,800 & 5,400 & 6,000 \\
\hline 3,500 & 4,200 & 4,900 & 5,600 & 6.300 & 7,000 \\
\hline 5,000 & 6,000 & 7,000 & 8,000 & 9,000 & 10,000 \\
\hline 6,000 & 7,200 & & 9,600 & 10,800 & 12,000 \\
\hline 1,000 & 1,200 & & & 1,800 & 2,000 \\
\hline 15,500 & 18,600 & 21,700 & 24,800 & 27,900 & 31,000 \\
\hline 120,000 & 144,000 & 168,000 & 192,000 & 216,000 & 240,000 \\
\hline
\end{tabular}

\begin{tabular}{rrrrrr}
80,000 & 96,000 & 112,000 & 128,000 & 144,000 & 160,000 \\
& & & & & \\
70,000 & 70,000 & 70,000 & 70,000 & 70,000 & 70,000 \\
3,000 & 3,000 & 3,000 & 3,000 & 3,000 & 3,000 \\
27,000 & 27,000 & 27,000 & 27,000 & 27,000 & 27,000 \\
100,000 & 100,000 & 100,000 & 100,000 & 100,000 & 100,000 \\
\hline$-20,000$ & $-4,000$ & 12,000 & 28,000 & 44,000 & 60,000 \\
\hline
\end{tabular}

Fuente Propia 


\section{Gráfico 1 Pronóstico de ingreso Bruto a Diferentes Niveles de ocupación}

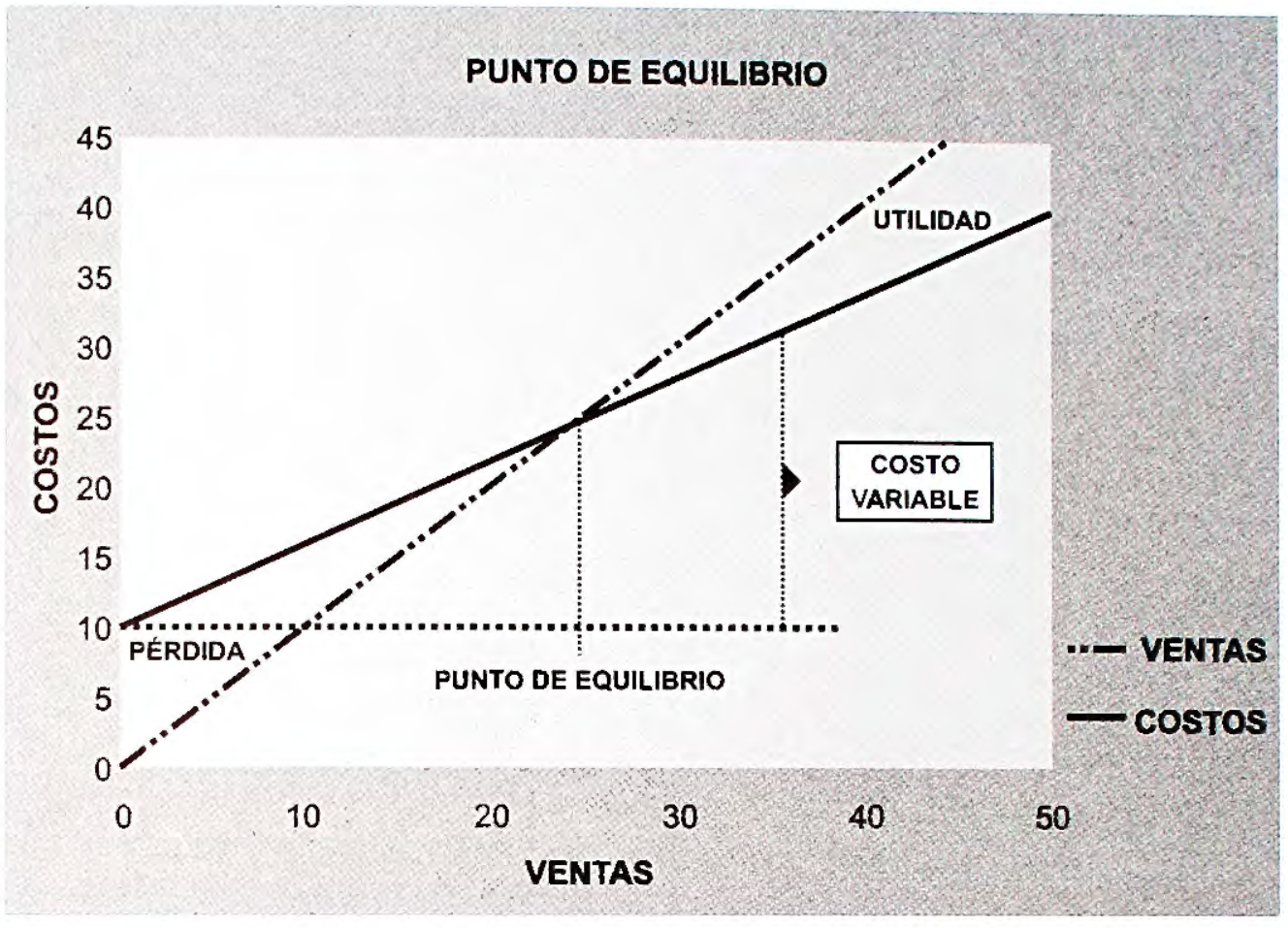

Fuente Propia

Del análisis tanto del cuadro número uno, como también del gráfico uno, el lector podrá tomar nota que el ingreso por concepto de ventas y los costos son atribuibles a los niveles de ocupación de la empresa hotelera, los mismos que fluctúan entre un $50,0 \%$ a un $100,0 \%$. Para efectos prácticos estos son los límites dentro de los cuales se puede esperar que opere un hotel producto del ejemplo.

\section{Análisis del punto de equilibrio}

Los datos de costo-volumen-utilidades pueden representarse gráficamente en la forma de una gráfica de equilibrio (gráfico número uno); el mismo indica la utilidad estimada que se obtendría con distintos valores de ventas. El gráfico también muestra el valor de las ventas mínimas para no tener pérdidas.

Un hotel si quiere ser eficiente debe de operar por encima del punto de equilibrio para poder reponer sus activos fijos (equipos) distribuir dividendos y contar con recursos necesarios para el caso de expandirse.
El punto de equilibrio puede derivarse matemáticamente de la siguiente manera:

Punto de equilibrio $=$

Costos fijos

$$
1 \text { - (costos variables/ventas) }
$$

Si aplicamos para cualquier nivel de ocupación de acuerdo con el cuadro número uno, veremos que el punto de equilibrio será el siguiente:

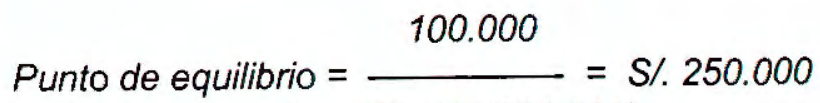

$$
1-(168.000 / 280.000)
$$

El lector podrá en función al cuadro número uno verificar el punto de equilibrio para los niveles de ocupabilidad y verá que el mismo es uno y sólo uno, para cualquiera de los niveles de ocupación bajo el supuesto implícito de que existe una proporcionalidad entre los costos variables y las ventas, manteniéndose el mismo nivel de costos fijos. 


\section{Significado de los patrones de punto de equilibrio}

La gráfica de equilibrio señala la actividad o tipo del negocio. Las compañias que incurren en costos fijos muy elevados, normalmente muestra un grafico com o el ilustrado en el gráfico número dos

Gráfico 2 Puntos de equilibrio

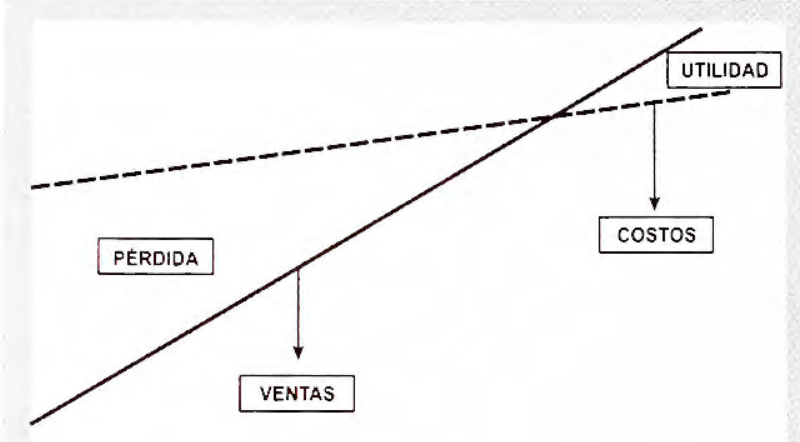

Fuente Propia

El objetivo primordial de una buena gerencia, con costos fijos elevados, tales como los hoteles es evidentemente la maximización de los ingresos. Los hoteles encuentran que es económicamente rentable ofrecer tarifas reducidas para las temporadas bajas siempre y cuando estos ingresos provenientes de las tarifas reducidas sobrepasen los costos incurridos. (Una mejor explicación es que los ingresos marginales, sean mayor a los costos marginales o variables).

\section{Análisis de equilibrio y decisiones respecto al cierre de una empresa hotelera}

Para ello es necesario el diferenciar entre los costos vivos y los costos extinguidos, los costos variables en general se deben considerar como costos vivos. Los costos fijos pueden caer dentro de cualquiera de estas dos categorias. Los costos vivos fijos aunque si bien es cierto que no varían con los cambios de los niveles de ocupabilidad de un hotel, representan gastos en efectivo por incurrirse, por ejemplo supervisión de equipos del hotel, servicios públicos. Los costos extinguidos fijos representan gastos o erogaciones efectuadas previamente y de los cuales quedan montos por ser derivados a los costos, como ejemplo de ello podemos citar el caso de las depreciaciones. De ello se desprende que la diferencia entre los ingresos de un hotel y sus costos vivos, equivale a la utilidad en efectivo. Si se analiza el cuadro número dos o el grafico número tres, se podrá diferenciar las utilidades contables y las utilidades en efectivo. Los datos contenidos en el cuadro número dos y el gráfico número tres señalan que el punto de equilibrio está al nivel de S/. 250.000 de ventas. El punto de cierre se encuentra a un nivel mucho más bajo $\mathrm{S} / .100 .000$ de las ventas. Si el hotel operara a niveles de venta por debajo de los S/. 1000.000, los costos vivos superarían a los ingresos en efectivo y sería aconsejable cerrar o reestructurar los ingresos, costos y gastos del hotel.

Cuadro 2 Proyección de Costos Vivos y Costos Extinguidos en diferentes niveles de ventas

\begin{tabular}{|c|c|c|c|c|c|}
\hline \multirow[b]{2}{*}{$\begin{array}{c}\text { Volumen de } \\
\text { ventas }\end{array}$} & \multirow{2}{*}{$\begin{array}{c}\text { Costos } \\
\text { incumidos } \\
\text { variables }\end{array}$} & \multicolumn{2}{|c|}{ Costos fijos } & \multicolumn{2}{|c|}{ Utilidad } \\
\hline & & vivos & extinguidos & $\begin{array}{l}\text { según } \\
\text { registro }\end{array}$ & $\begin{array}{c}\text { en } \\
\text { efectivo }\end{array}$ \\
\hline 200,000 & 120,000 & 40,000 & 60,000 & $-20,000$ & 40,000 \\
\hline 240,000 & 144,000 & 40,000 & 60,000 & $-4,000$ & 56,000 \\
\hline 280,000 & 168,000 & 40,000 & 60,000 & 12,000 & 72,000 \\
\hline 320,000 & 192,000 & 40,000 & 60,000 & 28,000 & 88,000 \\
\hline 360,000 & 216,000 & 40,000 & 60,000 & 44,000 & 104,000 \\
\hline 400,000 & 240,000 & 40,000 & 60,000 & 60,000 & 120,000 \\
\hline
\end{tabular}




\section{Gráfico 3 Proyección de Costos Vivos y Costos Extinguidos en diferentes niveles de ventas}

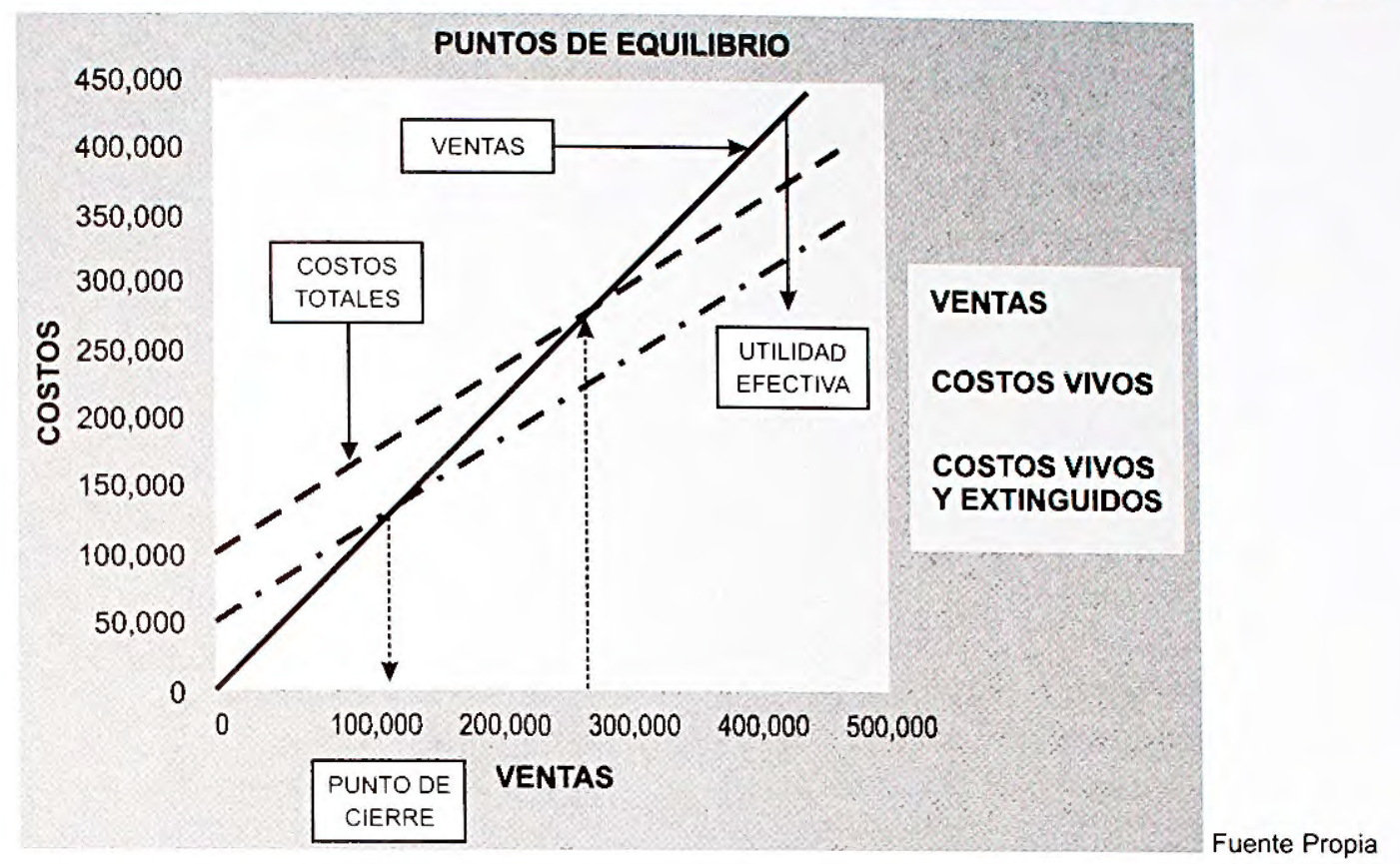

\section{Análisis de equilibrio y decisiones de expansión de una empresa hotelera.}

Los gráficos de equilibrio ayudan a revelar los cambios propuestos en la administración de un hotel, cuando el mismo se trata de expansiones en sus ventas, siempre y cuando la relación existente entre las ventas y los cotos variables no se vea afectada por los cambios.

Los datos presentados en el cuadro número uno han servido de base para ayudar a elaborar el gráfico número cuatro a efectos de mostrar una expansión de un hotel; para tales efectos un estudio de mercado revela que es posible el incrementar las ventas a S/.500.000,00 en un período de tiempo razonablemente corto, la solución propuesta para el hotel es aumentar su capacidad de prestación de servicios de $S / .400 .000,00$ a $S / .500 .000,00$ en ventas, pero a la vez también aumentarán los costos fijos en S/. $25.000,00$

Del análisis del gráfico se puede desprender, que si antes de la expansión el punto de equilibrio equivalía a un ingreso de $\mathrm{S} /$. $250.000,00 \mathrm{El}$ nuevo punto de equilibrio es de S/. $312.500,00$. Y ello como consecuencia del incremento en los costos fijos.

\section{Gráfico 4 Expansión en las ventas hoteleras}

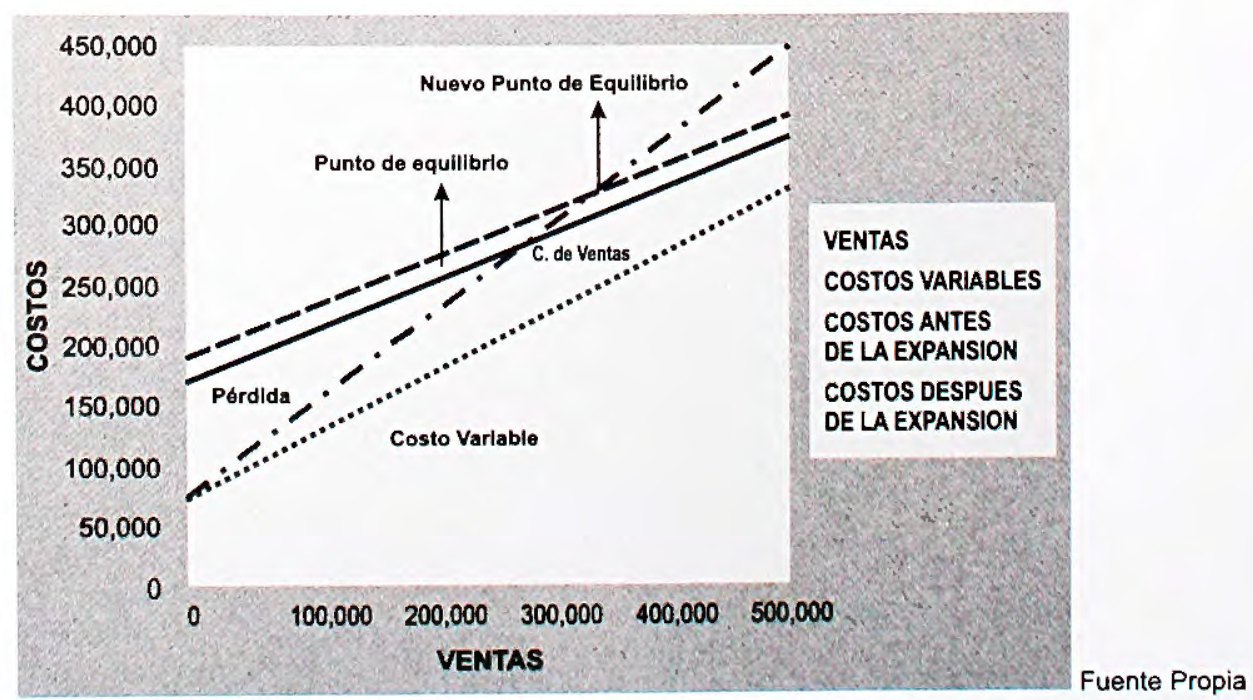




\section{Análisis del equilibrio y la variación en los precios}

El cambio en el precio del producto, asumiendo que la demanda que tiene una empresa hotelera es elástica, implicará que una disminución en sus tarifas, conllevará a un mayor incremento en la cantidad demandada, de tal suerte que el ingreso total por el rubro de los servicios hoteleros será mayor.

Lo anteriormente expuesto puede ilustrarse suponiendo que en una empresa hotelera, el precio promedio diario de sus servicios asciende a S/. 10,00 .00, el costo promedio variable por unidad de servicio es de S/. 6,00 y se venden 20.000.00 unidades de servicio; el gráfico número 5 muestra el margen de contribución que se obtendría para distintos niveles de venta en el caso de que aumente o baje dicho precio en un $10 \%$ en la actualidad el hotel esta vendiendo 16.000,00 unidades de servicio. Para poder obtener las mismas utilidades con un aumento del precio en un $10,0 \%$ solo deberán venderse $12.800,00$ unidades de servicio, en tanto que con una disminución del precio en un $10,0 \%$ deberán venderse $21.333,00$ unidades a efectos de evitar que bajen las utilidades a un valor menor al actual.

Un aumento en los precios disminuye el punto de equilibrio y una reducción del precio lo aumenta. La magnitud de los costos fijos influye en el grado de efecto que tienen los cambios del precio sobre los puntos de equilibrio. Este fenómeno se le conoce con el nombre de palanqueo de las operaciones.

Este efecto lo podemos ver con los siguientes costos fijos: S/. 20.000,00 S/. 30.000,00 $0 \mathrm{~S} / .40 .000,00$ (ver cuadro 3 y gráfico 5 )

\section{Cuadro 3 Ventas y Puntos de equilibrio}

\begin{tabular}{c|c|c|c|c|c|c}
\hline $\begin{array}{c}\text { Cantidad } \\
\text { Unidades } \\
\text { Vendidas }\end{array}$ & Precio & Ventas & C. variables & C. Fijos & T.costos & $\begin{array}{c}\text { Punto de } \\
\text { Equilibrio }\end{array}$ \\
\hline 21,333 & 9 & 191,997 & 127,998 & 30,000 & 157,998 & 90,000 \\
16,000 & 10 & 160,000 & 96,000 & 20,000 & 116,000 & 50,000 \\
12,800 & 11 & 140,800 & 76,800 & 10,000 & 86,800 & 22,000 \\
\hline
\end{tabular}

Gráfico 5 Efectos en los cambios de los precios

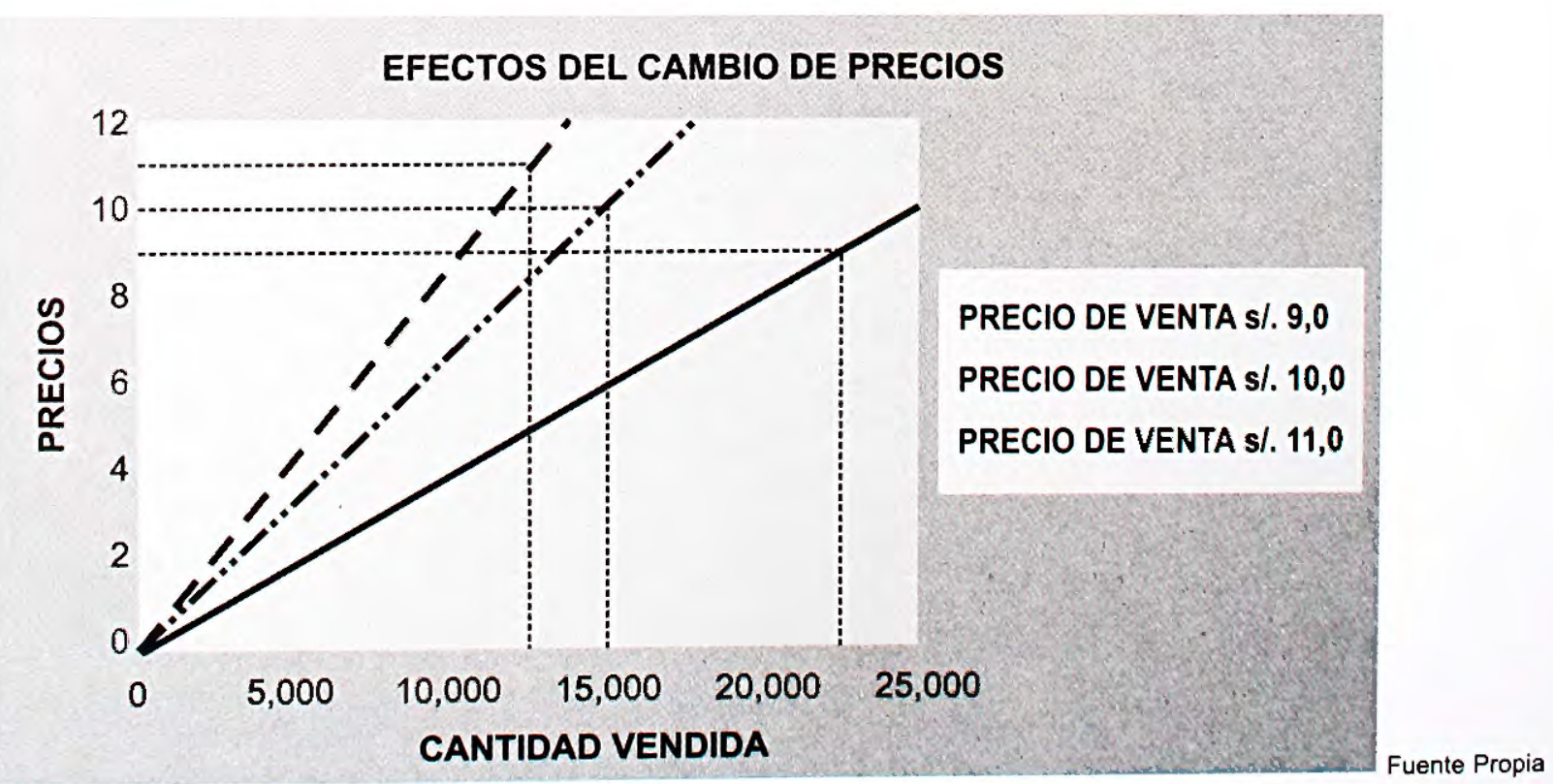




\section{Análisis de equilibrio y combinaciones de servicios en hotelería}

Dentro de la capacidad de planta de una empresa hotelera, esta puede aumentar el margen de contribución y las unidades de servicios producidos, y bajar el punto de equilibrio mediante una maximización en la combinación de los servicios vendidos. Ello implica que $\sin$ deteriorar la calidad total del servicio al cliente se venda más de los productos que reflejen un margen de contribución más alto en relación al precio. El lector podrá ilustrarse mejor si analiza el cuadro número cuatro

Cuadro 4 Análisis de equilibrio y combinaciones de servicios

\begin{tabular}{lccc}
\hline & Servicio & Servicio & Servicio \\
& $A$ & $B$ & $C$ \\
\hline Ventas & 100,000 & 120,000 & 400,000 \\
Costos Variables & 60,000 & 60,000 & 120,000 \\
Margen de contribucion & 40,000 & 60,000 & 280,000 \\
\hline Costos fijos & 15,000 & 15,000 & 15,000 \\
\hline Utilidad & 25,000 & 45,000 & 265,000 \\
Punto de equilibrio & 37,500 & 30,000 & 21,429 \\
\hline
\end{tabular}

Fuente Propia

El cuadro nos indica que conforme se combinen paulatinamente los servicios, el margen de contribución tiende a subir al igual que la utilidad neta y el punto de equilibrio tiende a bajar.

\section{Conclusiones}

Resulta de particular importancia para las empresas hoteleras el cuantificar constantemente sus puntos de equilibrio, dado que el mercado en la que se desenvuelven es sumamente errático y depende de muchas variables exógenas, es decir de variables que escapan al control de la empresa.
La fuente primigenia para efectuar un análisis de Costo-Volumen-Utilidades, son los registros contables, de donde se pueden extraer los datos necesarios para elaborar este tipo de análisis. Sin embargo el analista que realice este tipo de análisis debe de efectuar estudios previos de correlación y análisis de dispersión de estos. Luego de que estos análisis reflejen un grado de confiabilidad a efectos de poder proyectar las ventas y los costos, es necesario efectuar un análisis cualitativo de estas cifras, porque en una economía globalizada los gustos $y$ tendencias son sumamente cambiantes, por lo que ignorar lo que está aconteciendo en el mundo y guiarnos exclusivamente de las tendencias estadísticas nos llevaría muchas veces a tomar decisiones equivocadas en perjuicio de la empresa hotelera.

Los costos fijos en hotelería son relativamente altos, en tal sentido, cuanto más alto sea el margen de retribución de los costos variables en relación a las ventas, más bajo será el punto de equilibrio.

Con respecto a los costos fijos, para elaborar un análisis como el expuesto, es recomendable que se tenga en cuenta que los costos fijos tienen una capacidad de cambio de corto plazo, por lo que se recomienda evaluar los activos fijos de una empresa con mucho cuidado.

Finalmente el lector deberá tener en consideración, que un aumento en los precios disminuye el punto de equilibrio $y$ una reducción del precio lo aumenta. La magnitud de los costos fijos influye en el grado de efecto que tienen los cambios del precio sobre los puntos de equilibrio. Este fenómeno se le conoce con el nombre de palanqueo de las operaciones.

José Marsano Delgado Instituto de Investigación

La Escuela Profesional de Turismo y Hoteleria Universidad de San Martín de Porres, Lima 